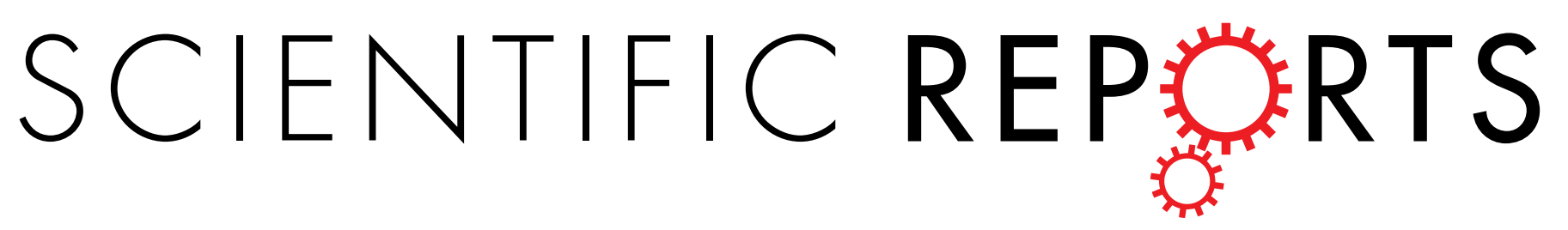

\title{
OPEN Author Correction: First Evidence of Coherent Bands of Strong Turbulent Layers Associated with High-Wavenumber Internal-Wave Shear in the Upstream Kuroshio
}

\author{
Takeyoshi Nagai ${ }^{1}$, Daisuke Hasegawa², Takahiro Tanaka², Hirohiko Nakamura ${ }^{3}$, \\ Eisuke Tsutsumi ${ }^{4}$, Ryuichiro Inove ${ }^{5}$ \& Toru Yamashiro ${ }^{6}$
}

Correction to: Scientific Reports https://doi.org/10.1038/s41598-017-15167-1, published online 06 November 2017

This Article contains an error in the Acknowledgments section.

'We thank Capt. Uchiyama, C/O Azuma, Prof. Nishina, Prof. Kobari, Prof. Matsuno, Prof. Senju, Dr. Li, crews, and all the participants of the R/T/V Kagoshima-maru cruise KG1616. T.N. thanks Hancyk, Dr. Wolk at RSI, Dr. Li, Seki, and Yoshii at JAC, Dr. Kokubu at NORTEK, Rutka at STS, support from OMIX (MEXT KAKENHI JP16H01590) and SKED ("Study of Kuroshio Ecosystem Dynamics for Sustainable Fisheries" funded to FRA from MEXT). D.H., T.T., and R.I. thank OMIX (MEXT KAKENHI) JP15H05818. H.N., and E.T. thank OMIX (MEXT KAKENHI) JP15H05821'.

should read:

'We thank Capt. Uchiyama, C/O Azuma, Prof. Nishina, Prof. Kobari, Prof. Matsuno, Prof. Senju, Dr. Li, crews, and all the participants of the R/T/V Kagoshima-maru cruise KG1616. T.N. thanks Hancyk, Dr. Wolk at RSI, Dr. Li, Seki, and Yoshii at JAC, Dr. Kokubu at NORTEK, Rutka at STS, support from OMIX (MEXT KAKENHI JP16H01590). D.H., T.T., and R.I. thank OMIX (MEXT KAKENHI) JP15H05818. H.N., and E.T. thank OMIX (MEXT KAKENHI) JP15H05821.

(i) Open Access This article is licensed under a Creative Commons Attribution 4.0 International (c) License, which permits use, sharing, adaptation, distribution and reproduction in any medium or format, as long as you give appropriate credit to the original author(s) and the source, provide a link to the Creative Commons license, and indicate if changes were made. The images or other third party material in this article are included in the article's Creative Commons license, unless indicated otherwise in a credit line to the material. If material is not included in the article's Creative Commons license and your intended use is not permitted by statutory regulation or exceeds the permitted use, you will need to obtain permission directly from the copyright holder. To view a copy of this license, visit http://creativecommons.org/licenses/by/4.0/.

(C) The Author(s) 2018

\footnotetext{
${ }^{1}$ Tokyo University of Marine Science and Technology, Department of Ocean Sciences, Tokyo, 108-8477, Japan. ${ }^{2}$ Tohoku National Fisheries Research Institute, Japan Fisheries Research and Education Agency, Fisheries Oceanography and Resources Department, Shiogama, Miyagi, 985-0001, Japan. ${ }^{3}$ Kagoshima University, Faculty of Fisheries, Kagoshima, 890-0056, Japan. 'Kyushu University, Research Institute for Applied Mechanics, Kasuga, Fukuoka, 816-8580, Japan. ${ }^{5}$ Research and Development Center for Global Change, Japan Agency for Marine-Earth Science and Technology, Yokosuka, 237-0061, Japan. ${ }^{6}$ Graduate School of Science and Engineering, Kagoshima University, Kagoshima, 890-0065, Japan. Daisuke Hasegawa, Takahiro Tanaka, Hirohiko Nakamura, Eisuke Tsutsumi, Ryuichiro Inove and Toru Yamashiro contributed equally to this work. Correspondence and requests for materials should be addressed toT.N. (email: tnagai@kaiyodai.ac.jp)
} 